\title{
Bulk electronic, elastic, structural, and dielectric properties of the Weyl semimetal TaAs
}

\author{
J. Buckeridge, ${ }^{*}$ D. Jevdokimovs, C. R. A. Catlow, and A. A. Sokol \\ University College London, Kathleen Lonsdale Materials Chemistry, Department of Chemistry, \\ 20 Gordon Street, London WC1H OAJ, United Kingdom
}

(Received 14 December 2015; revised manuscript received 11 February 2016; published 8 March 2016)

\begin{abstract}
We present results of electronic structure calculations of the bulk properties of the Weyl semimetal TaAs. The emergence of Weyl (massless) fermions in TaAs, due to its electronic band structure, is indicative of a new state of matter in the condensed phase that is of great interest for fundamental physics and possibly new applications. Many of the physical properties of the material, however, are unknown. We have calculated the structural parameters, dielectric function, elastic constants, phonon dispersion, electronic band structure, and Born effective charges using density functional theory within the generalized gradient approximation, including spin-orbit coupling where necessary. Our results provide essential information on the material; and our calculations agree well with the relatively small number of experimental data available. Moreover, we have determined the relative stability of the ground state body-centered tetragonal phase with respect to other common binary phases as a function of pressure at the athermal limit, predicting a transition to the $\mathrm{CsCl}$ cubic structure at $23.3 \mathrm{GPa}$. Finally, we have determined the band structure using an unbiased hybrid density functional that includes $25 \%$ exact exchange, in order to refine the previously determined positions in $k$ space of the Weyl points.
\end{abstract}

DOI: 10.1103/PhysRevB.93.125205

\section{INTRODUCTION}

Weyl fermions are massless chiral spin- $\frac{1}{2}$ particles that are allowed solutions of Dirac's equation [1-3]. Although theoretically possible, the demonstration of their existence has remained elusive until quite recently, where they have been shown to be emergent properties of so-called "Weyl semimetals," of which the archetypal example is the binary system TaAs [4]. Measurements of the Fermi surface and band structure using angle resolved photoemission spectroscopy (ARPES), coupled with first principles calculations, have shown conclusively the Weyl points and surface Fermi arcs that are indicative of the fermions in TaAs [5-9] (as well as the related materials $\mathrm{TaP}, \mathrm{NbAs}$, and $\mathrm{NbP}$ [10-12]; other systems studied are $\mathrm{SrSi}_{2}$ [13] and $\mathrm{Na}_{3} \mathrm{Bi}$ [14], while a particular photonic crystal design has been proposed [15] to allow Weyl fermions).

As well as representing a novel topological state of matter and therefore being of great interest for fundamental physics, TaAs has been shown to exhibit negative magnetoresistance [16] and high carrier mobilities [17], which may have substantial device implications. Relatively little is known, however, about its elastic, structural, optical, and dielectric properties. Measurements have shown it to be diamagnetic and nonmetallic $[18,19]$, with a low Seebeck coefficient and high thermal conductivity [20].

In ambient conditions, TaAs stabilizes in a body-centered tetragonal phase $\left(I 4_{1} m d\right.$, space group no. 109) [18,21], with other stoichiometries possible and probably present in small traces in single-crystal samples of TaAs [20,22]. The Ta and As atoms have six nearest neighbors in a trigonal prismatic coordination, with the prisms lying lengthwise perpendicular to the $c$ axis, stacked in alternate orientations rotated by $90^{\circ}$ [see Fig. 1(a)]. The relative stability of this phase with respect to other common binary phases, either six-coordinated

\footnotetext{
*j.buckeridge@ucl.ac.uk
}

octahedrally, or eight- or four-coordinated, is not understood at present.

In this paper, we have calculated the structural, elastic, dielectric, lattice vibrational, optical, and electronic properties of TaAs using density functional theory (DFT), including spin-orbit coupling effects where necessary. Our results provide crucial information on the physical properties of this material for future experimental design. Our calculated phonon frequencies are in excellent agreement with Raman measurements [23]. We have studied the stability of the $I 4_{1} m d$ phase relative to common binary phases and have calculated their pressure dependence. We find that the hexagonal NiAs phase $\left(P 6_{3} / m m c\right.$, no. 194) is next nearest in enthalpy, but as the pressure is increased the cubic $\mathrm{CsCl}$ structure $(P m \overline{3} m$, no. 221), which is metallic with no Weyl points present, stabilizes and becomes the most favorable at pressures above $23.3 \mathrm{GPa}$. The zinc blende phase is highly unfavorable at all pressures studied. We have also calculated the band structure using a hybrid-DFT approach that includes $25 \%$ screened exact exchange (which corrects for the self-interaction error in DFT) in order to refine the positions of the Weyl points in reciprocal space. Our refinement is consistent with Fermi arc measurements using ARPES and provides useful information for comparison for future experiments and calculations.

The paper is now structured as follows. In Sec. II we discuss our calculation techniques in detail, in Sec. III we present our results, and in Sec. IV we give a summary of our work and the resulting conclusions.

\section{METHODS}

All our DFT calculations were carried out using the VASP code [24-27], utilizing the solids-corrected PerdewBurke-Ernzerhof (PBEsol) GGA exchange-correlation (XC) functional $[28,29]$ with the projector augmented wave (PAW) method [30] to model the interaction between core and valence electrons (with five valence electrons for both Ta and As atoms). Using the implementation in VASP [31], spin-orbit 

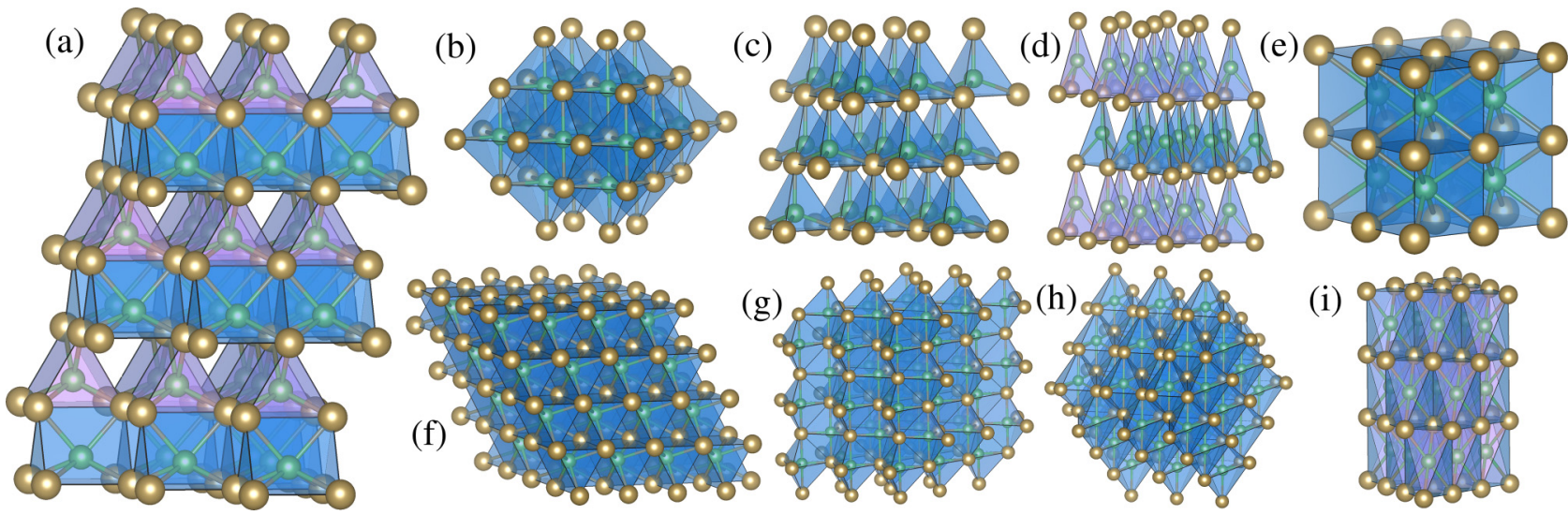

(i)

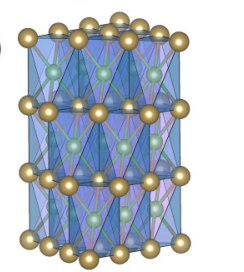

FIG. 1. Crystal structure of the phases studied: (a) The ground state (body-centered tetragonal $I 4_{1} m d$ ), (b) rock salt (cubic $F m \overline{3} m$ ), (c) zinc blende (cubic $F \overline{4} 3 m$ ), (d) wurtzite (hexagonal $P 6_{3} m c$ ), (e) $\mathrm{CsCl}$ (cubic $P m \overline{3} m$ ), (f) NiAs (hexagonal $P 6_{3} / m m c$ ), (g) 5-5 (five-coordinated orthorhombic $\mathrm{Cmcm})$, (h) six-coordinated orthorhombic $(P \mathrm{mmn})$, (i) monoclinic $(\mathrm{C} 2 / \mathrm{m})$ structure. The structures are shown as being built up of As-centered polyhedra [blue/purple (gray)], with As atoms in green (dark gray) and Ta atoms in gold (light gray).

interactions were included where necessary for electronic structure calculations (the interaction was found to have a negligible effect on the structural, dielectric, and vibrational properties and on enthalpy differences between phases and so was not included in those cases). When determining the electronic band structure we have used the screened hybrid exchange correlation functional HSE06 [32], where 25\% exact exchange is included, which is known to reduce appropriately the self-interaction error present in DFT to a satisfactory degree in many cases [33-36].

To avoid the problem of Pulay stress, the four atom primitive unit cell of TaAs was optimized at a series of different volumes with a $400 \mathrm{eV}$ plane wave cutoff and a $9 \times 9 \times 9$ MonkhorstPack [37] $k$-point mesh, which provided convergence in the total energy up to $10^{-4} \mathrm{eV}$. The resulting energy-volume data were fitted to the Murnaghan equation of state to derive the equilibrium lattice constant and bulk modulus. This procedure was also followed for the other phases studied, with an $18 \times$ $18 \times 18 k$-point mesh for the cubic phases and an $18 \times 18 \times$ $9 k$-point mesh for all other cases. All force calculations were deemed converged when the change in total force on each ion per iteration was less than $10^{-2} \mathrm{eV} \AA^{-1}$.

The Born effective charges and piezoelectric tensor components were determined using the linear response approach implemented in VASP [38], while the dielectric function was determined using a summation over calculated conduction bands [39]. Phonon frequencies of TaAs were calculated using the frozen phonon approach, where the dynamical matrix is derived by displacing atoms from their equilibrium positions and calculating the resulting forces, thus giving the numerical force constants. In order to carry out this procedure, the primitive cell geometry was relaxed in the manner described above but deemed converged when the change in total force on each ion per iteration was less than $10^{-4} \mathrm{eV} \AA^{-1}$, so that more accurate forces could be computed. The phonon dispersion was calculated by displacing atoms appropriately in a $4 \times 4 \times 4$ (256 atoms) expansion of the primitive unit cell and constructing the dynamical matrix, as implemented in the post-processing program PHONOPY [40]. The splitting between the transverse optical (TO) and longitudinal optical
(LO) modes was determined using a nonanalytical correction term [41-43].

\section{RESULTS}

\section{A. Structural and elastic properties}

Our calculated structural properties, using the PBEsol functional, of TaAs in its ground state phase (body-centred tetragonal $I 4_{1} m d$ ) are presented in Table I, in comparison with experimentally determined values. We have also included our results using the HSE06 hybrid functional. In both cases the results are in agreement with experiment within $\leqslant 0.5 \%$. Also shown in Table I are the structural parameters of the other binary structures studied (see Fig. 1): rock salt (cubic $F m \overline{3} m$, no. 225 ), zinc blende (cubic $F \overline{4} 3 m$, no. 216), wurtzite (hexagonal $P 6_{3} m c$, no. 186), $\mathrm{CsCl}$ (cubic $P m \overline{3} m$, no. 221), NiAs (hexagonal $P 6_{3} / m m c$, no. 194), and three structures that were discovered serendipitously as local minima close to those studied previously. The first was found in a minimum close to that of wurtzite, but with five-coordinated ions rather than four, where each ion sits in the center of a triangle of coplanar ions of the opposite charge, occupying alternate triangles in the hexagonal layers of the wurtzite structure, with two more ions completing a trigonal bipyramidal building block [see Fig. 1(g)]. This phase has an orthorhombic primitive cell ( $\mathrm{Cmcm}$, no. 63, but we give the conventional hexagonal cell parameters in Table I) and has been observed from global optimization calculations on a number of systems where it has been dubbed the "5-5" structure [44-46]. Close in the energy landscape to the 5-5 phase we determined a six-coordinated orthorhombic phase (Pmmn, no. 59), with close-packed distorted octahedra as building blocks, [Fig. 1(h)]. The third structure was found close to the zinc blende phase, consisting of trigonally distorted octahedra in a monoclinic $(C 2 / m$, no. 12) cell [see Fig. 1(i)]. The enthalpy difference $(\Delta H)$ between these phases and the ground state is included, as is the bulk modulus $(B)$ and its derivative $\left(B^{\prime}\right)$.

At zero applied pressure, in the athermal limit, it is valid to equate the total energy with enthalpy and compare the energies per atom of the different phases to assess their relative 
TABLE I. Calculated lattice parameters $a$ and $c$, internal coordinate $u$, bulk modulus $B$ and its derivative $B^{\prime}$, and enthalpy above the ground state phase $\Delta H$ (in the athermal limit) for TaAs in the binary phases $I 4_{1} m d$ (ground state), rock salt, zinc blende, wurtzite, CsCl, NiAs, 5-5, six-coordinated orthorhombic $(\mathrm{Pmmn})$, and monoclinic $(\mathrm{C} 2 / \mathrm{m})$. The calculations have been performed using the PBEsol functional except where indicated (HSE06). Experimental values are included for comparison where available.

\begin{tabular}{|c|c|c|c|c|c|c|c|}
\hline & $a(\AA)$ & $b(\AA)$ & $c(\AA)$ & $u$ & $B(\mathrm{GPa})$ & $B^{\prime}$ & $\Delta H\left(\mathrm{~kJ} \mathrm{~mol}^{-1}\right)$ \\
\hline$I 4_{1} m d$ & 3.429 & 3.429 & 11.583 & 0.418 & 192.3 & 4.6 & \\
\hline$I 4_{1} m d$ (HSE06) & 3.423 & 3.423 & 11.609 & 0.418 & 202.5 & 4.6 & \\
\hline$I 4_{1} m d$ (Experiment $)^{\mathrm{a}}$ & 3.4368 & 3.4368 & 11.6442 & 0.4177 & & & \\
\hline Rock salt & 5.206 & 5.206 & 5.206 & & 175.8 & 4.2 & 114.0 \\
\hline Zinc blende & 5.735 & 5.735 & 5.735 & & 113.1 & 4.2 & 230.2 \\
\hline Wurtzite & 2.994 & 2.994 & 9.269 & 0.280 & 163.0 & 5.3 & 147.6 \\
\hline $\mathrm{CsCl}$ & 3.178 & 3.178 & 3.178 & & 202.3 & 4.1 & 49.4 \\
\hline $\mathrm{NiAs}$ & 3.337 & 3.337 & 7.023 & & 187.3 & 4.0 & 15.1 \\
\hline $5-5$ & 4.377 & 4.377 & 5.104 & & 135.9 & 4.8 & 140.3 \\
\hline Pmmn & 3.274 & 4.919 & 4.221 & 0.143 & 164.6 & 4.8 & 111.9 \\
\hline$C 2 / m$ & 5.016 & 3.227 & 4.546 & & 168.6 & 4.5 & 69.3 \\
\hline
\end{tabular}

${ }^{\mathrm{a}}$ Ref. [18]

stability. Comparing each phase with the ground state, we find that the closest in enthalpy is the NiAs phase, being $15.1 \mathrm{~kJ} \mathrm{~mol}^{-1}$ less stable. The NiAs structure also contains six coordinated ions in trigonal prisms but differs from the ground state in that alternating layers of prisms are collinear and alternate the orientation of their square faces along the layers, so that the triangular channels seen in the ground state phase are not present [see Figs. 1(a) and 1(f)]. The next lowest energy structures are $\mathrm{CsCl}$, where the ions are eight coordinated, followed by the six coordinated monoclinic $C 2 / m$, orthorhombic, and rock salt phases. Lower coordinated structures are at least $140.3 \mathrm{~kJ} \mathrm{~mol}^{-1}$ less stable than the ground state, and hence unfavorable, reflecting the preference for higher coordination (we note that they may become more favorable under negative pressure, e.g. due to chemical expansion). Wurtzite is considerably more stable than zinc blende, which at first is surprising as the two structures only differ in their close packing. On closer inspection, however, we see that the relaxed wurtzite phase displays significant

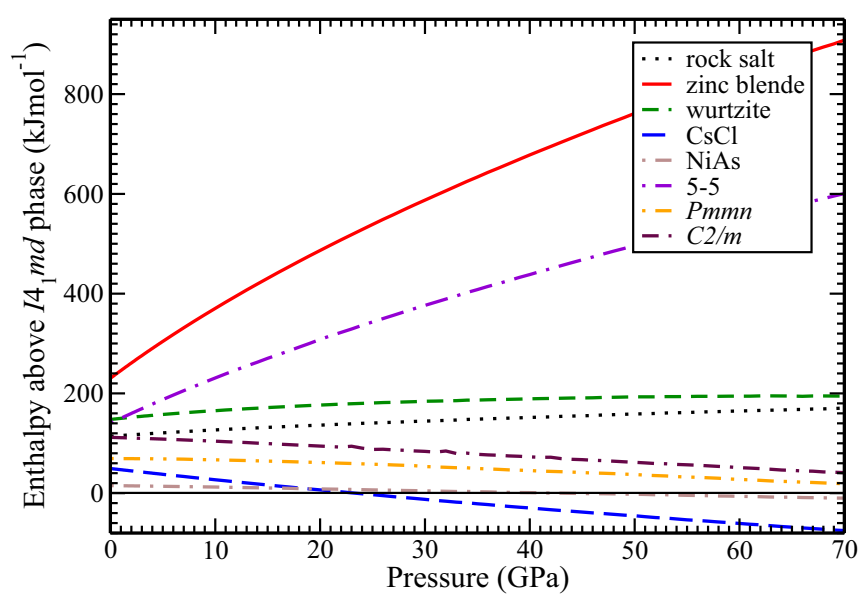

FIG. 2. Enthalpy of each TaAs phase relative to that of the initial ground state phase $\left(I 4_{1} m d\right)$, shown as a function of applied pressure. The phases studied are specified by their common names where available; otherwise the space group is given. elongation along the $c$ direction [see Fig. 1(d)], allowing an increase in Ta-As bond length $(2.604 \AA$ in wurtzite and ground state phase vs $2.483 \AA$ in zinc blende) to equal that in the ground state phase, while also resulting in relatively close second nearest neighbors, thus stabilizing the structure relative to zinc blende.

We have also calculated the pressure dependence of the relative stability of each binary phase and present our results in Fig. 2. For nonzero pressure we compare the enthalpies of each phase. As the pressure $p$ increases, the NiAs phase gradually becomes closer in enthalpy to the $I 4_{1} m d$ phase while the $\mathrm{CsCl}$ phase rapidly becomes closer until $\mathrm{CsCl}$ becomes the lowest enthalpy phase at $p=23.3 \mathrm{GPa}$. This result reflects the preference for higher coordination at higher pressure. The relative stabilisation of the NiAs phase, as well as the Pmmn and $C 2 / m$ phases, which have the same coordination as the $I 4_{1} m d$ phase (six), is a consequence of the close packing of the polyhedra. Indeed, we see that rock salt becomes less favorable with applied pressure, as this phase has corner-sharing octahedra and therefore no close packing.

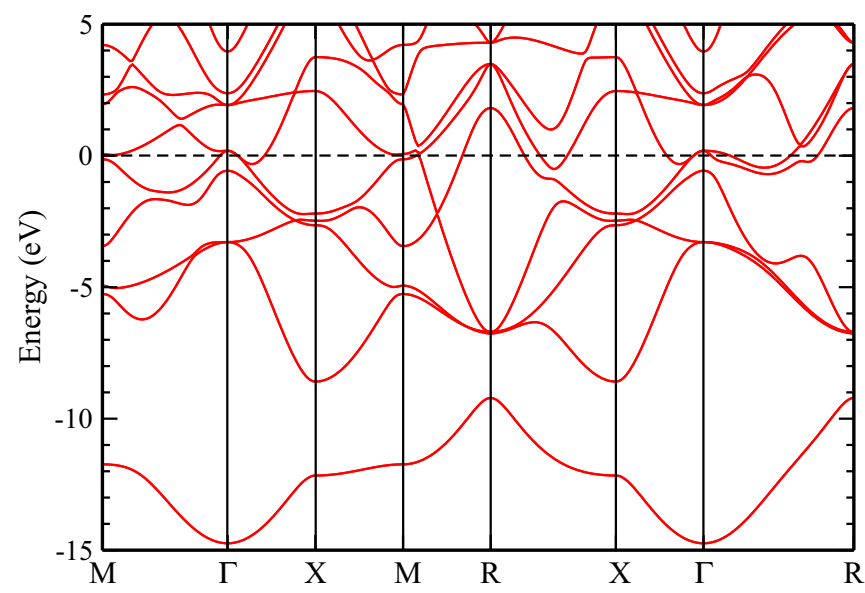

FIG. 3. Electronic energy band structure of $\mathrm{TaAs}$ in the $\mathrm{CsCl}$ phase (cubic $\operatorname{Pm} \overline{3} m$ ) at ambient pressure. The Fermi energy is used as the reference. 
TABLE II. Calculated elastic tensor components $\mathrm{C}_{i j}$, Born effective charges $Z_{i i}^{*}$ for each element, and piezoelectric tensor components $d_{i j}$ of TaAs in its ground state phase. e is the electronic charge.

\begin{tabular}{lc}
\hline \hline $\mathrm{C}_{11}, \mathrm{C}_{12}, \mathrm{C}_{13}(\mathrm{GPa})$ & $310.3,178.0,138.0$ \\
$\mathrm{C}_{33}, \mathrm{C}_{44}, \mathrm{C}_{66}(\mathrm{GPa})$ & $260.7,93.0,197.8$ \\
$Z_{11}^{*}, Z_{22}^{*}, Z_{33}^{*} \mathrm{Ta}(\mathrm{e})$ & $7.57,13.57,1.25$ \\
$Z_{11}^{*}, Z_{22}^{*}, Z_{33}^{*} \mathrm{As}(\mathrm{e})$ & $-1.62,-19.80,-1.19$ \\
$d_{15}, d_{31}, d_{33}\left(\mathrm{Cm}^{-2}\right)$ & $4.95,0.81,-1.89$ \\
\hline \hline
\end{tabular}

The five- and four-coordinated phases become less stable with pressure, as expected. The calculated band structure, including spin-orbit coupling, of $\mathrm{TaAs}$ in the $\mathrm{CsCl}$ phase (at ambient pressure) is shown in Fig. 3. We see that the system is metallic, with no evidence of the presence of Weyl points (as expected for a structure with inversion symmetry).

The elastic tensor components $\mathrm{C}_{i j}$ for TaAs are presented in Table II. The values confirm the mechanical stability of the structure, which, for example, is comparable with that of rutile and anatase $\mathrm{TiO}_{2}[47,48]$.

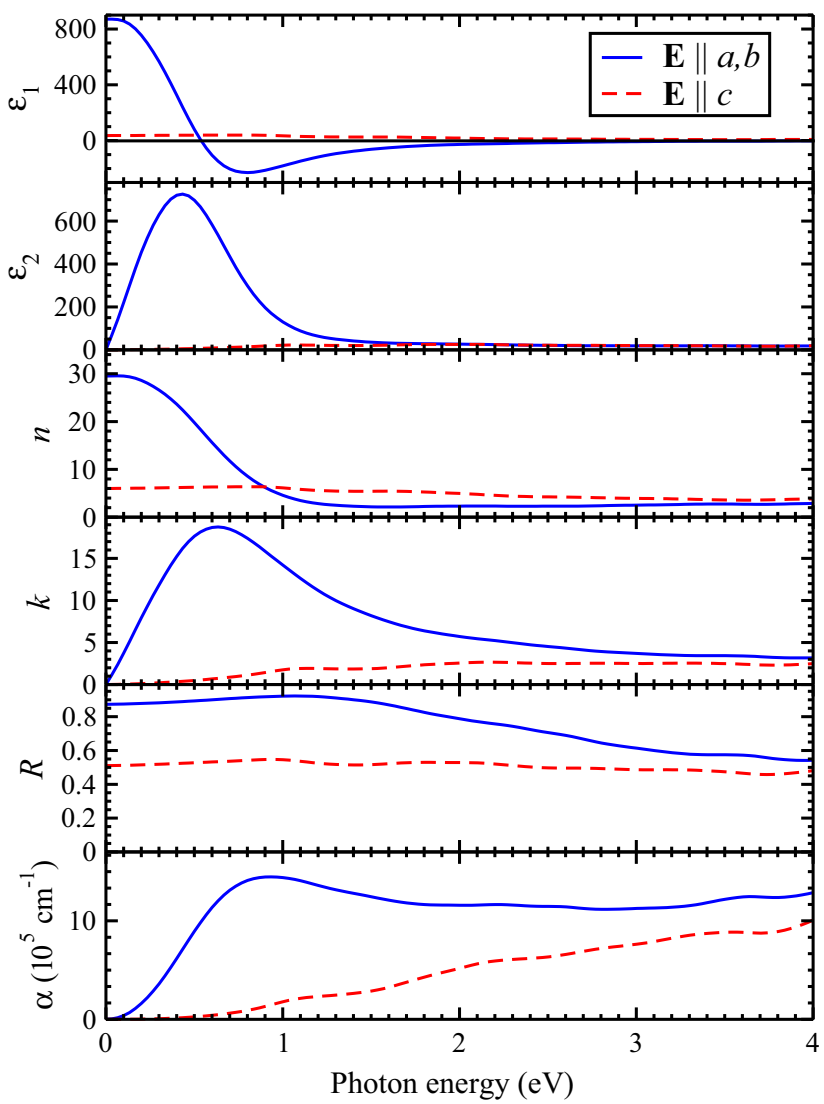

FIG. 4. Dielectric function and derived optical parameters of TaAs as a function of photon energy: real $\left(\varepsilon_{1}\right)$ and imaginary $\left(\varepsilon_{2}\right)$ parts of the dielectric function, real $(n)$ and imaginary $(k)$ parts of the complex refractive index, reflectance $R$, and absorption coefficient $\alpha$. The cases where the impinging electric field $\mathbf{E}$ is parallel to the $a$ or $b$ directions (solid blue line) and parallel to $c$ (red dashed line) are shown.

\section{B. Dielectric and vibrational properties}

Using the linear response technique [38], we have calculated the Born effective charges $Z^{*}$ of TaAs which are presented in Table II. As the structure comprises edge-sharing trigonal prisms, one square face of which lies in the $a b$ plane, the polarizability for displacements along $a$ and $b$ differs, resulting in three different $Z^{*}$ values. The primitive cell consists of two atoms of each species, which lie in alternating layers of prisms that stack in the $c$ direction [see Fig. 1(a)]. The $Z_{11}^{*}$ and $Z_{22}^{*}$ values swap depending on the layer the atom lies in, which is a consequence of the relative rotation of the prisms by $\pi / 2$ radians between the layers. Calculated piezoelectric tensor components $d_{i j}$ are also given in Table II. The values are significant and indicate the high polarity of bonding and strong coupling between the polarization and strain in the system.

The calculated dielectric function $\left(\tilde{\varepsilon}=\varepsilon_{1}+i \varepsilon_{2}\right)$ is shown in Fig. 4 as a function of photon energy, along with the derived complex index of refraction $\tilde{n}=n+i k\left(\tilde{n}^{2}=\tilde{\varepsilon}\right)$, the reflectance $R=|(\tilde{n}-1) /(\tilde{n}+1)|^{2}$, and optical absorption $\alpha=4 \pi k / \lambda$ (where $\lambda$ is the photon wavelength). Due to the tetragonal symmetry, the optical properties differ for the cases of the impinging oscillating electric field $\mathbf{E}$ being parallel to the $a$ or $b$ directions or parallel to the $c$ direction. In the visible

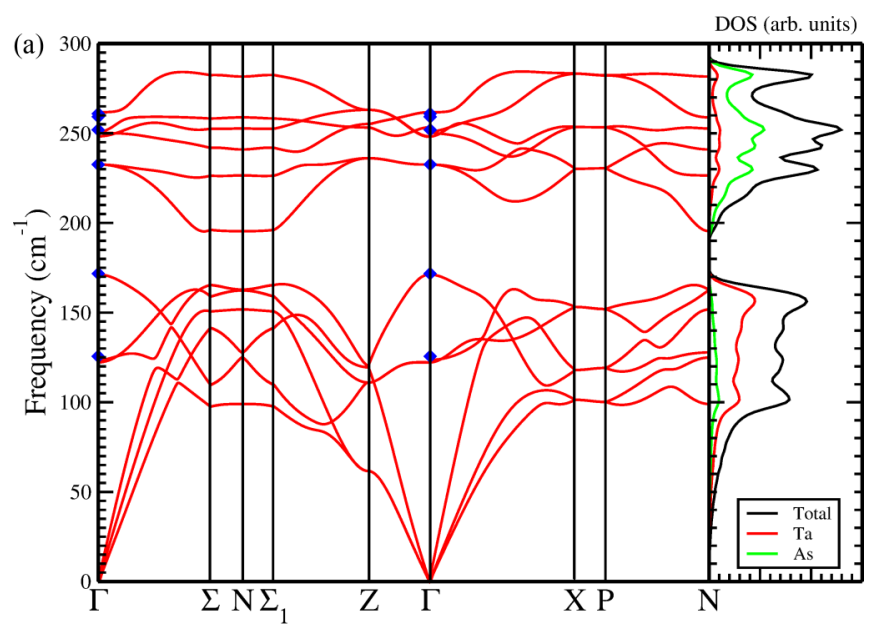

(b)

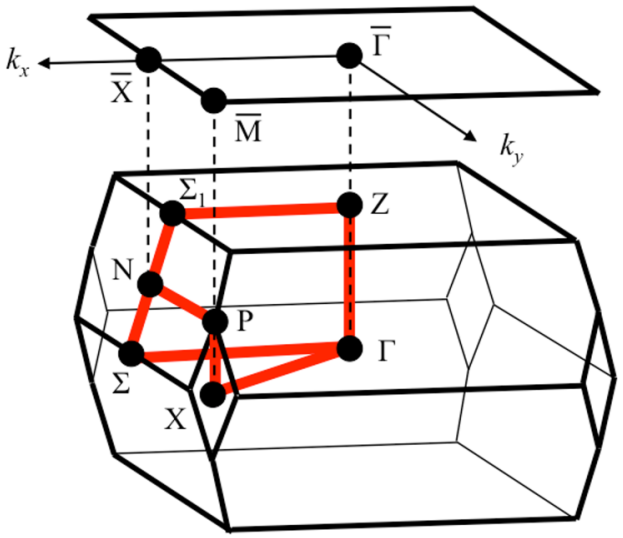

FIG. 5. (a) Phonon dispersion and density of states (DOS) of TaAs, including projections onto the ion types in the unit cell, in the Brillouin zone. Inelastic Raman scattering measurements from Ref. [23] (blue diamonds) are included for comparison. (b) Schematic of the $I 4_{1} m d$ bulk and (001) surface first Brillouin zones. 

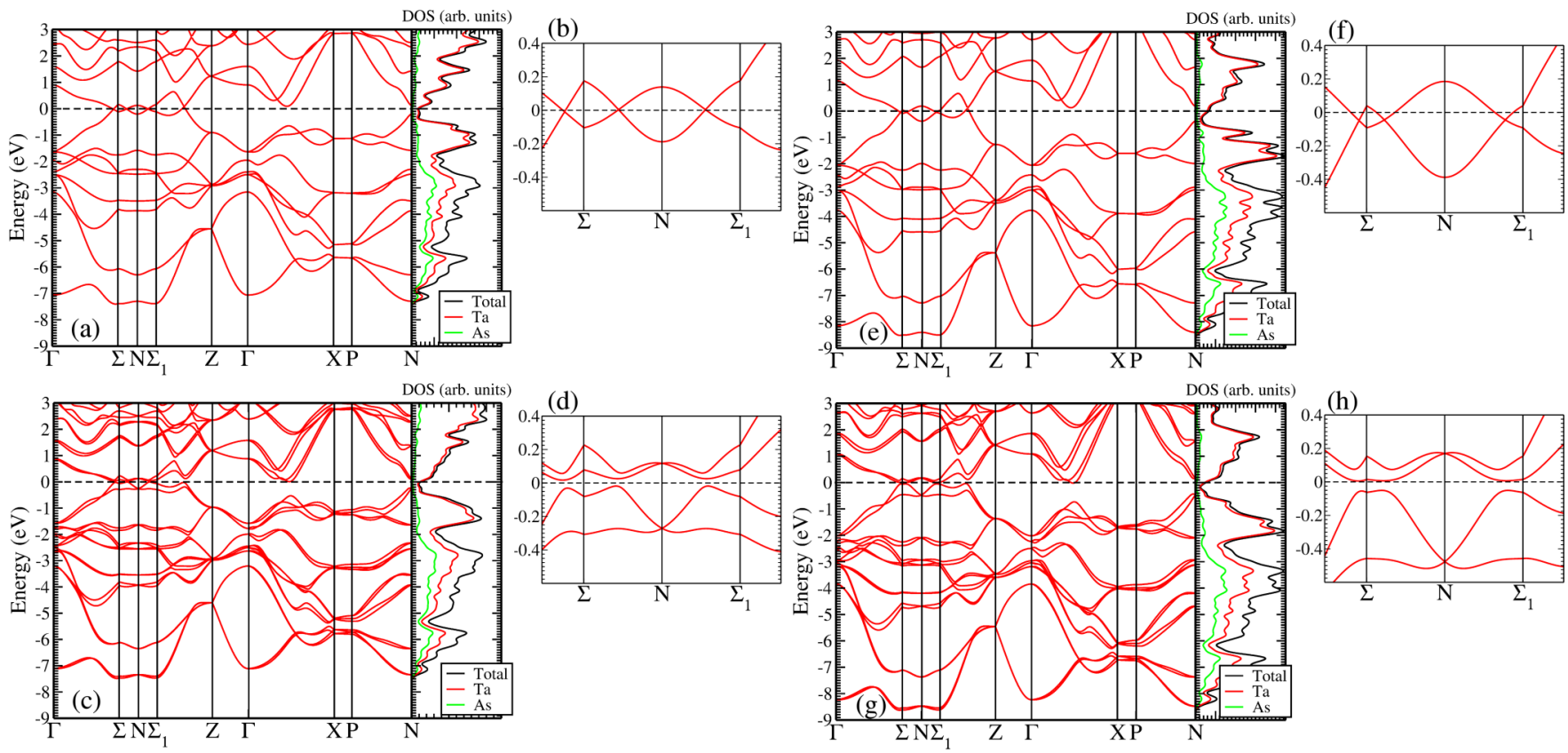

FIG. 6. Electronic band structure of TaAs along the high-symmetry path through the Brillouin zone, calculated using PBEsol (a) and (b), PBEsol including spin-orbit coupling (SOC) (c) and (d), HSE06 (e) and (f), HSE06 including SOC (g) and (h). The Fermi energy is used as the reference. The zoom-ins (b) and (f) show the band crossings near the $N$ point, which collapse to the Weyl points away from high symmetry paths in the BZ when SOC is included [(d) and (h)].

spectrum, the material absorbs and reflects light, as expected for a semimetal and in agreement with the image of single crystals given in Ref. [4]. The properties are similar to those seen for the narrow-gap systems $\mathrm{PbS}$ and $\mathrm{PbTe}$ [47]. Taking the long-wavelength limit, we find that the high-frequency dielectric constants are $\varepsilon_{11}=871, \varepsilon_{33}=36$. These values would be further enhanced due to thermal excitations which are not included in our simulations.

We have calculated the phonon dispersion using the frozen phonon approach, and our results are shown in Fig. 5, including the phonon density of states (DOS) and its projection on the individual ions. For comparison, we include the results of Raman measurements from Ref. [23], with which our results are in excellent agreement. We note that Liu et al. in Ref. [23] also calculated the phonon dispersion, using the PBE rather than the PBEsol functional. Our results demonstrate that PBEsol is more appropriate as it leads to values much closer to experiment, a consequence of more accurate interatomic forces resulting from this functional [49-51]. From the phonon DOS shown in Fig. 5 we find that the acoustic modes are distributed over both ion species, while the optical modes are split into lower frequency modes mainly concentrated on the heavier (and less polarizable) Ta ions and higher frequency modes mainly concentrated the lighter As ions. The linear dispersion observed along $\Sigma-N-\Sigma_{1}$ and along $X-P$ is probably a result of an insufficient sampling of the first Brillouin zone in these regions.

\section{Electronic band structure and Weyl points}

We present our calculated band structures in Fig. 6, using PBEsol with [Figs. 6(a) and 6(b)] and without [Figs. 6(c) and 6(d)] spin-orbit coupling effects, and HSE06 with
[Figs. 6(e) and 6(f)] and without [Figs. 6(g) and 6(h)] spin-orbit coupling effects. When spin-orbit effects are not included, there are points in the band structure where the top valence band (VB) and bottom conduction band (CB) cross, with near linear dispersion (shown more clearly in Figs. 6(b) and 6(f). As shown in Ref. [4], beyond the high-symmetry directions these points extend into nodal rings on the mirror planes parallel to the $k_{z}$ direction in the Brillouin zone (BZ), which in turn reduce to points (the Weyl points) when spin-orbit coupling effects are included. These points, where the bands display linear dispersion, are characteristic of the massless Weyl fermions and lead to features such as Fermi arcs on the (001) surface that can be experimentally measured, the existence of which has confirmed TaAs as a Weyl semimetal [5-9]. Comparing the PBEsol band structure with that determined using HSE06, we see that the crossing points occur at different energies relative to the Fermi level, which implies that the positions of the loops and hence the Weyl points will differ according to the DFT approach used.

By calculating the $\mathrm{CB}$ and $\mathrm{VB}$ dispersion in the full $\mathrm{BZ}$, we have determined the positions of the Weyl points in reciprocal space and their projections onto the (001) surface, which has been studied experimentally using ARPES [5-9]; Fig. 7 and Table III compare our PBEsol and HSE06 results with those of Huang et al. [4], who used PBE. The high-symmetry points in the BZ of the surface are indicated by letters with bars. There are some small differences between the results from the two approaches. By convention, the Weyl points in TaAs (and related systems with the same crystal structure) are divided into two types, according to their $k_{z}$ value in reciprocal space: $\mathrm{W} 1\left(k_{z}=2 \pi / c\right)$ and $\mathrm{W} 2\left(k_{z} \neq 2 \pi / c\right)$ [4]. On the (001) surface, the W1 points lie furthest from $\bar{\Gamma}$ (see Fig. 7). Using HSE06, we find that the W1 points are slightly closer 


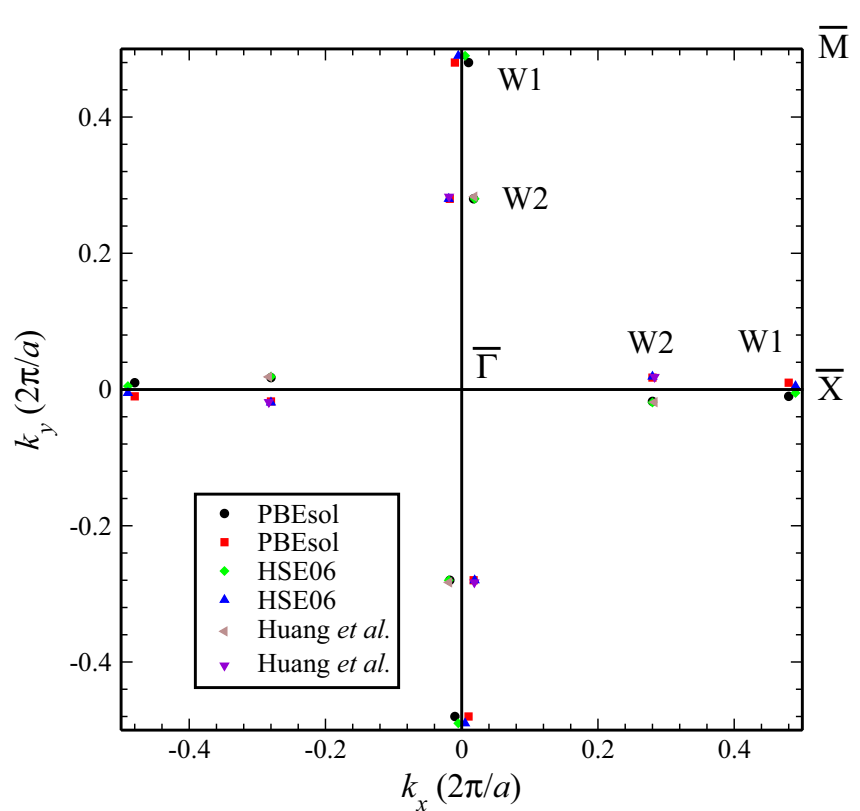

FIG. 7. Calculated positions of the Weyl points in the Brillouin zone of TaAs projected onto the (001) surface, determined using the PBEsol (black squares and red circles) and HSE06 (green diamonds and blue upwards-pointing triangles) functionals. For comparison the results of Huang et al. [4] are shown (brown left-pointing and purple downwards-pointing triangles). (The different colors for each functional indicate points of opposite chirality. The Weyl points are labeled as W1 and W2 according to their $k_{z}$ value in reciprocal space (see text for details).

to the zone edge, which would result in narrower but more pronounced Fermi arcs [12]. Our results using PBEsol agree well with those using PBE [4-7,12], but the more pronounced Fermi arcs associated with $\mathrm{W} 1$ that would be seen if the HSE06 Weyl points were assumed to be correct would be in better agreement with the experimental results $[5-7,12]$. We stress that the difference is small, and it may be the case that it is within the experimental error. Nevertheless, our HSE06 results provide a useful comparison for future measurements of Fermi arcs on TaAs (001). The resulting coordinates of the W1 point projected onto the (001) surface are $\boldsymbol{k}_{W 2}=\left(0.009 \AA^{-1}, 0.899 \AA^{-1}\right)$.

For the W2 point, from HSE06 we find that the coordinates projected to the (001) surface are $\boldsymbol{k}_{W 2}=$ $\left(0.035 \AA^{-1}, 0.587 \AA^{-1}\right)$, in excellent agreement with the
TABLE III. Calculated positions $\boldsymbol{k}$ of the W1 and W2 Weyl points in the bulk Brillouin zone (BZ) of TaAs and the energy difference between them $(\Delta E)$, determined using PBEsol and HSE06 and compared with the PBE results from Ref. [4] and Ref. [5]. The BZ positions are given in units of $2 \pi / a(2 \pi / c)$ for $k_{x}$ and $k_{y}\left(k_{z}\right)$. Only the W2 point has a nontrivial $k_{z}$ value (see text).

\begin{tabular}{lccc}
\hline \hline & $\mathrm{W} 1\left(k_{x}, k_{y}\right)$ & $\mathrm{W} 2\left(k_{x}, k_{y}, k_{z}\right)$ & $\Delta E(\mathrm{meV})$ \\
\hline PBEsol & $(0.01,0.48)$ & $(0.0175,0.28,0.58)$ & 13 \\
HSE06 & $(0.005,0.49)$ & $(0.019,0.32,0.58)$ & 16 \\
PBE [4,5] & $(0.0072,0.5173)$ & $(0.0185,0.2831,0.6000)$ & 14 \\
\hline \hline
\end{tabular}

calculations of Huang et al. [4] and within the experimental error from ARPES measurements given in Ref. [6] (the PBEsol results are almost identical, see Fig. 7). From HSE06 we determine a difference in energy between the Weyl points of $16 \mathrm{meV}$, in good agreement with the $14 \mathrm{meV}$ value given in Ref. [5].

\section{SUMMARY/CONCLUSIONS}

To summarize, we have calculated the structural, elastic, dielectric, lattice vibrational, and electronic properties of the Weyl semimetal TaAs using DFT. Our results agree well with available experiment and previous calculations where available but provide much needed physical data previously unknown about this increasingly important material. We have determined the pressure dependence of the ground state phase in comparison with a variety of common binary phases, finding that the $\mathrm{NiAs}$ and $\mathrm{CsCl}$ phases are closest in energy, with the $\mathrm{CsCl}$ phase, which is metallic with no Weyl points, becoming more favorable at $p=23.3 \mathrm{GPa}$. Moreover, we have calculated the band structure using a hybrid density functional, which provides a refinement of the position of the Weyl points in reciprocal space which will be of use for comparison with future higher resolution ARPES measurements.

\section{ACKNOWLEDGMENTS}

The authors acknowledge funding from EPSRC Grant No. EP/K016288/1. The authors also acknowledge the use of the UCL Legion High Performance Computing Facility (Legion@UCL) and associated support services and the national supercomputer ARCHER through membership of the UK's High End Computing (HEC) Materials Chemistry Consortium, which is funded by EPSRC Grant No. EP/L000202, in the completion of this work.
[1] P. A. M. Dirac, Proc. R. Soc. London A 117, 610 (1928).

[2] H. Weyl, Z. Phys. 56, 330 (1929).

[3] P. B. Pal, Am. J. Phys. 79, 485 (2011).

[4] S.-M. Huang, S.-Y. Xu, I. Belopolski, C.-C. Lee, G. Chang, B. Wang, N. Alidoust, G. Bian, M. Neupane, C. Zhang, S. Jia, A. Bansil, H. Lin, and M. Z. Hasan, Nat. Commun. 6, 7373 (2015).

[5] S.-Y. Xu, I. Belopolski, N. Alidoust, M. Neupane, G. Bian, C. Zhang, R. Sankar, G. Chang, Z. Yuan, C.-C. Lee, S.-M.
Huang, H. Zheng, J. Ma, D. S. Sanchez, B. Wang, A. Bansil, F. Chou, P. P. Shibayev, H. Lin, S. Jia, and M. Z. Hasan, Science 349, 613 (2015).

[6] L. X. Yang, Z. K. Liu, Y. Sun, H. Peng, H. F. Yang, T. Zhang, B. Zhou, Y. Zhang, Y. F. Guo2, M. Rahn, D. Prabhakaran, Z. Hussain, S.-K. Mo, C. Felser, B. Yan, and Y. L. Chen, Nat. Phys. 11, 728 (2015).

[7] B. Q. Lv, H. M. Weng, B. B. Fu, X. P. Wang, H. Miao, J. Ma, P. Richard, X. C. Huang, L. X. Zhao, G. F. Chen, 
Z. Fang, X. Dai, T. Qian, and H. Ding, Phys. Rev. X 5, 031013 (2015).

[8] B. Q. Lv, N. Xu, H. M. Weng, J. Z. Ma, P. Richard, X. C. Huang, L. X. Zhao, G. F. Chen, C. E. Matt, F. Bisti, V. N. Strocov, J. Mesot, Z. Fang, X. Dai1, T. Qian, M. Shi, and H. Ding, Nat. Phys. 11, 724 (2015).

[9] B. Q. Lv, S. Muff, T. Qian, Z. D. Song, S. M. Nie, N. Xu, P. Richard, C. E. Matt, N. C. Plumb, L. X. Zhao, G. F. Chen, Z. Fang, X. Dai, J. H. Dil, J. Mesot, M. Shi, H. M. Weng, and H. Ding, Phys. Rev. Lett. 115, 217601 (2015).

[10] H. Weng, C. Fang, Z. Fang, B. A. Bernevig, and X. Dai, Phys. Rev. X 5, 011029 (2015).

[11] Y. Sun, S.-C. Wu, and B. Yan, Phys. Rev. B 92, 115428 (2015).

[12] Z. K. Liu, L. X. Yang, Y. Sun, T. Zhang, H. Peng, H. F. Yang, C. Chen, Y. Zhang, Y. F. Guo, D. Prabhakaran, M. Schmidt, Z. Hussain, S.-K. Mo, C. Felser, B. Yan, and Y. L. Chen, Nat. Mater. 15, 27 (2016).

[13] S.-M. Huang, S.-Y. Xu, I. Belopolski, C.-C. Lee, G. Chang, T.-R. Chang, B. Wang, N. Alidoust, G. Bian, M. Neupane, D. Sanchez, H. Zheng, H.-T. Jeng, A. Bansil, T. Neupert, H. Lin, and M. Z. Hasan, Proc. Natl. Acad. Sci. U.S.A. 113, 1180 (2016).

[14] J. Xiong, S. K. Kushwaha, T. Liang, J. W. Krizan, M. Hirschberger, W. Wang, R. J. Cava, and N. P. Ong, Science 350, 413 (2015).

[15] L. Lu, L. Fu, J. D. Joannopoulos, and M. Soljacic, Nat. Photon. 7, 294 (2013).

[16] X. Huang, L. Zhao, Y. Long, P. Wang, D. Chen, Z. Yang, H. Liang, M. Xue, H. Weng, Z. Fang, X. Dai, and G. Chen, Phys. Rev. X 5, 031023 (2015).

[17] C. Zhang, Z. Yuan, S. Xu, Z. Lin, B. Tong, M. Z. Hasan, J. Wang, C. Zhang, and S. Jia, arXiv:1502.00251.

[18] S. Furuseth, K. Selte, and A. Kjekshus, Acta Chem. Scand. 19, 95 (1965).

[19] Y. Liu, Z. Li, L. Guo, X. Chen, Y. Yuan, F. Liu, S. Prucnal, M. Helm, and S. Zhou, J. Magn. Magn. Mater. 408, 73 (2016).

[20] B. Saparov, J. E. Mitchell, and A. S. Sefat, Supercond. Sci. Technol. 25, 084016 (2012).

[21] H. Boller and E. Parthé, Acta Cryst. 16, 1095 (1963).

[22] J. J. Murray, J. B. Taylor, L. D. Calvert, Y. Wang, E. J. Gabe, and J. G. Despault, J. Less Common Met. 46, 311 (1976).

[23] H. W. Liu, P. Richard, Z. D. Song, L. X. Zhao, Z. Fang, G.-F. Chen, and H. Ding, Phys. Rev. B 92, 064302 (2015).

[24] G. Kresse and J. Hafner, Phys. Rev. B 47, 558 (1993).

[25] G. Kresse and J. Hafner, Phys. Rev. B 49, 14251 (1994).

[26] G. Kresse and J. Furthmüller, Comput. Mater. Sci. 6, 15 (1996).
[27] G. Kresse and J. Furthmüller, Phys. Rev. B 54, 11169 (1996).

[28] J. P. Perdew, K. Burke, and M. Ernzerhof, Phys. Rev. Lett. 77, 3865 (1996).

[29] J. P. Perdew, A. Ruzsinszky, G. I. Csonka, O. A. Vydrov, G. E. Scuseria, L. A. Constantin, X. Zhou, and K. Burke, Phys. Rev. Lett. 100, 136406 (2008).

[30] P. E. Blöchl, Phys. Rev. B 50, 17953 (1994).

[31] D. Hobbs, G. Kresse, and J. Hafner, Phys. Rev. B 62, 11556 (2000).

[32] J. Heyd, G. E. Scuseria, and M. Ernzerhof, J. Chem. Phys. 124, 219906 (2006).

[33] D. O. Scanlon and G. W. Watson, J. Mater. Chem. 22, 25236 (2012).

[34] A. Walsh, A. B. Kehoe, D. J. Temple, G. W. Watson, and D. O. Scanlon, Chem. Commun. 49, 448 (2013).

[35] D. O. Scanlon, J. Buckeridge, C. R. A. Catlow, and G. W. Watson, J. Mater. Chem. C 2, 3429 (2014).

[36] J. Buckeridge, K. T. Butler, C. R. A. Catlow, A. J. Logsdail, D. O. Scanlon, S. A. Shevlin, S. M. Woodley, A. A. Sokol, and A. Walsh, Chem. Mater. 27, 3844 (2015).

[37] H. J. Monkhorst and J. D. Pack, Phys. Rev. B 13, 5188 (1976).

[38] M. Gajdoš, K. Hummer, G. Kresse, J. Furthmüller, and F. Bechstedt, Phys. Rev. B 73, 045112 (2006).

[39] B. Adolph, J. Furthmüller, and F. Bechstedt, Phys. Rev. B 63, 125108 (2001).

[40] A. Togo, F. Oba, and I. Tanaka, Phys. Rev. B 78, 134106 (2008).

[41] R. M. Pick, M. H. Cohen, and R. M. Martin, Phys. Rev. B 1, 910 (1970).

[42] P. Giannozzi, S. de Gironcoli, P. Pavone, and S. Baroni, Phys. Rev. B 43, 7231 (1991).

[43] X. Gonze and C. Lee, Phys. Rev. B 55, 10355 (1997).

[44] J. Schön and M. Jansen, Comput. Mater. Sci. 4, 43 (1995).

[45] H. Haas and M. Jansen, Angew. Chem. Int. Ed. 38, 1909 (1999).

[46] J. C. Schön, T. Dinges, and M. Jansen, Z. Naturforsch. B 61, 650 (2006).

[47] D. R. Lide, editor, CRC Handbook of Chemistry and Physics, 89th edition (CRC Press, Boca Raton, FL, 2008).

[48] M. Iuga, G. Steinle-Neumann, and J. Meinhardt, Eur. Phys. J. B 58, 127 (2007).

[49] J. M. Skelton, S. C. Parker, A. Togo, I. Tanaka, and A. Walsh, Phys. Rev. B 89, 205203 (2014).

[50] J. Buckeridge, D. O. Scanlon, T. D. Veal, M. J. Ashwin, A. Walsh, and C. R. A. Catlow, Phys. Rev. B 89, 014107 (2014).

[51] J. Buckeridge, D. O. Scanlon, A. Walsh, C. R. A. Catlow and A. A. Sokol, Phys. Rev. B 87, 214304 (2013). 